\title{
The effect of daily chlorhexidine baths on nosocomial meticillin-resistant Staphylococcus aureus infection in MRSA-colonized patients admitted to general wards
}

\author{
Willis $\mathrm{GC}^{1}$, Ooi $\mathrm{ST}^{1}$, Foo $\mathrm{ML}^{2}$, Ong $\mathrm{PL}^{2}$, Tan $\mathrm{BC}^{2}$, Chen $\mathrm{YM}^{2}$, $\mathrm{Li} \mathrm{DL}^{2}$, Chng $\mathrm{FLC}^{2}$, \\ 1. Department of Medicine, Khoo Teck Puat Hospital, Singapore \\ 2. Department of Nursing, Khoo Teck Puat Hospital, Singapore \\ 3. Department of Nursing, Yishun Community Hospital, Singapore
}

doi: 10.3396/IJIC.v11i4.026.15

\begin{abstract}
Compared to the Intensive Care Unit setting, relatively little data has been published on the benefits of daily Chlorhexidine gluconate (CHG) baths in infection prevention on general wards. To contribute to our knowledge of this infection prevention strategy we report three years of cumulative data from a 550-bed general hospital in Singapore. Our hospital infection control policy mandates Meticillin-resistant Staphylococcus aureus (MRSA) entry-screening for all cases admitted to hospital. Positive cases are isolated or cohorted with appropriate contact precautions. In addition positive cases receive daily 4\% $\mathrm{CHG}$ baths throughout their stay. We compare the rates of hospital-acquired MRSA (HA-MRSA) infection in the cohort that screened MRSA-positive (and hence received daily CHG baths) with the cohort in whom MRSA was not detected and hence did not receive CHG baths. Of 4598 screen-positive cases only 4 developed HA-MRSA infection. This compares to 36 cases of HA-MRSA infection among 5391 patients who we estimate were undetected MRSA-carriers, either because they missed screening, or screened false-negative, or acquired MRSA during their hospital stay. MRSA-colonized patients receiving daily $\mathrm{CHG}$ baths while in hospital were significantly less likely to develop HA-MRSA infection (OR = 0.129; $\mathrm{P}=0.0001 ; 95 \% \mathrm{Cl} 0.046-0.36$; NNT $=172)$. We conclude that General Ward patients who screen MRSA-positive on admission and who receive daily $\mathrm{CHG}$ baths throughout hospitalization have a significantly lower rate of HA-MRSA infection compared to patients who screen MRSA-negative and who do not receive such $\mathrm{CHG}$ baths.
\end{abstract}

Keywords: Meticillin-resistant Staphylococcus aureus; Chlorhexidine; Anti infective agents, local; Baths; Wards, general

\section{Corresponding Author}

Dr Christopher Willis

Department of Medicine, Khoo Teck Puat Hospital, 90 Yishun Central, Singapore 768828

Email: willis.george@alexandrahealth.com.sg 


\section{Introduction}

Compared to the Intensive Care Unit (ICU) setting, relatively little has been published on the benefits of daily chlorhexidine gluconate (CHG) baths in infection prevention on general wards. To contribute to our knowledge of this infection prevention strategy we report three years of cumulative data from a 550-bed general hospital in Singapore. We compare hospitalacquired Meticillin-resistant Staphylococcus aureus (HA-MRSA) infection rates between two cohorts, one receiving daily $\mathrm{CHG}$ baths, the other not receiving such baths.

\section{Background}

There have been a fair number of studies demonstrating the beneficial effect of administering daily $\mathrm{CHG}$ baths to patients in the ICU setting. This effect has included a significant reduction in healthcare-associated bloodstream infections (HA-BSI), a finding which has been confirmed in a meta-analysis of 12 such studies. ${ }^{1}$ In addition to reducing HA-BSI, this strategy has also been shown to significantly reduce the transmission (acquisition) of multi-drug-resistant organisms (MDRO) in the ICU setting. ${ }^{2,3}$ By contrast, much less data is available on this issue for patients in the setting of a general ward. One study reports a significant reduction in Clostridium difficile infection with the use of hospital-wide daily CHG baths. ${ }^{4}$ Another study showed a significant reduction in HA-MRSA infections and HA-VRE (Vancomycin-resistant Enterococcus) infections using daily $\mathrm{CHG}$ bathing but failed to show a reduction in HA-C. difficile infections. ${ }^{5}$ We examine our accumulated data for the years 2011, ${ }^{6} 2012$ and 2013 to determine the effect on HA-MRSA infection for general ward patients receiving daily CHG baths.

\section{Methods}

Khoo Teck Puat Hospital is a 550-bed general hospital that opened in mid-2010 to serve the northern sector of Singapore. We do not provide services for paediatrics, obstetrics and gynaecology, organ transplantation, cardiac surgery, or haematology/oncology. As part of infection control policy all patients admitted to the hospital are screened on entry for MRSA-colonization by three-site nasal, axillae, and groin swabbing. One swab is used for the nares and one for the axillae/groin regions. The two swabs are then processed as one specimen. Culture method using ChromAgar $^{\mathrm{TM}}$ was used until July 2011 from which time batched PCR (Roche LightCycler ${ }^{\mathrm{TM}}$ ) was used in order to reduce the time to results. Sensitivity and specificity of PCR for MRSA detection was determined to be $92 \%$ and $99 \%$ respectively.

Khoo Teck Puat Hospital general wards each have 32 beds. These consist of two single rooms each with their own toilet and shower area, and six 5-bed cubicles with the 5 patients in each cubicle sharing one toilet and shower area located within the cubicle. Our infection control policy mandates daily $4 \%$ CHG baths for all patients admitted to hospital pending results of MRSA screening. Those who screen MRSA-positive will be isolated or cohorted and managed with appropriate contact precautions which consist of apron, gloves, and hand-hygiene. In addition they continue daily CHG bathing throughout their hospital stay whereas those who test MRSA-negative stop. The rationale for this policy is simply to reduce the overall MRSA burden on the patient's skin. This is not an attempt at decolonization.

Our patient profile consists of about $80 \%$ ambulatory and $20 \%$ non-ambulatory cases. Among the ambulatory cases $3.4 \%$ are MRSA-carriers whereas this figure is $18 \%$ among non-ambulatory cases. Overall given the much greater proportion of ambulatory cases this category actually makes up about $43 \%$ of MRSA carriers as compared to $57 \%$ of carriers being nonambulatory.

Non-ambulatory MRSA-positive patients are bathed by nursing staff using $40 \mathrm{ml}$ of $4 \%$ CHG applied over the whole body (except the face) using a clean disposable washcloth. After 3 minutes on the skin the $\mathrm{CHG}$ is rinsed off. Ambulatory patients are provided with a bottle of $4 \% \mathrm{CHG}$ and instructed to use the same procedure when they take their daily shower. Should a patient have skin sensitivity to $\mathrm{CHG}$ (contact dermatitis or anaphylaxis) the alternative antiseptic agent Octenidine is used instead for daily bathing. However this is a rare problem. This protocol for antiseptic bathing is set out in a poster on each ward and nursing staff are very familiar with it. The nursing managers of the general wards were approached individually and asked an open-ended question about the situations in which $\mathrm{CHG}$ bathing was administered 
in their particular ward. They each demonstrated familiarity with our infection control policy of using daily CHG baths for MRSA-positive patients. For bedbound patients compliance is high (estimated $>90 \%$ ) as nursing staff are the ones administering the baths. For ambulatory cases compliance in providing the patient with bottles of $4 \% \mathrm{CHG}$ and explaining its usage is also high, but it is difficult to verify its actual use by the patient.

In this report we compare the rates (Table I) and types (Table II) of MRSA infection occurring during hospitalization (HA-MRSA) for two groups: Those who screened positive for MRSA on admission and who therefore received daily $\mathrm{CHG}$ baths throughout their hospital stay; and those in whom MRSA was not detected on admission and who therefore did not receive daily $\mathrm{CHG}$ baths.

\section{Results}

Table I shows the actual numbers of patients admitted in 2011, 2012 and 2013 together with the results of their admission screening for MRSA. The table also presents the number of cases which progressed to HA-MRSA infection in: 1) the cohort that was MRSA-positive on admission screening (and therefore received daily CHG baths); and in 2) the cohort that tested MRSAnegative or who missed screening and hence did not receive daily $\mathrm{CHG}$ baths. The method for determining the rates of infection for the two cohorts is discussed below.
Colonization with MRSA is the usual sequence before HA-MRSA infection can occur in a particular patient. For those patients who screened MRSA-negative on admission but who subsequently developed HA-MRSA infection there are two possible scenarios. Some may have been carriers who tested falsely negative on admission (given PCR detection sensitivity of 92\%) and some may have become colonized with MRSA through cross-transmission or in some cases possibly through antibiotic exposure. In order to determine the MRSA acquisition rates for General Ward patients we conducted entry-exit swabs on 861 consecutive general ward MRSA negative patients at discharge. This was done over the 4-month period from December 2010 until the end of March 2011. This revealed the average MRSA acquisition rate during hospitalization to be $5 \%$. Using this information we can estimate (Figure 1) the number of patients who were unidentified MRSA carriers. This group includes some among those who missed screening and some who screened MRSAnegative on admission but who actually were already colonized or who became colonized while in hospital. It is this whole group of unidentified MRSA carriers who are at risk for progression to actual MRSA infection. The grand total of cases estimated to be undetected carriers and not getting daily CHG baths because they were either unscreened or tested MRSA-negative on entry is 5391 (see description beneath Figure 1).

Cumulative results over the years 2011,2012 and 2013 reveal the following: 4598 cases tested MRSA-positive

Table I. Number of patients admitted in 2011till 2013 and the results of their admission screening for MRSA

\begin{tabular}{|c|c|c|c|c|c|c|c|}
\hline \multirow{3}{*}{ Year } & \multirow{3}{*}{$\begin{array}{c}\text { Total Cases } \\
\text { Admitted }\end{array}$} & \multirow{3}{*}{$\begin{array}{l}\text { Total Cases } \\
\text { Screened }\end{array}$} & \multicolumn{2}{|c|}{ Total Cases } & \multirow{3}{*}{$\begin{array}{l}\text { Total not } \\
\text { screened }\end{array}$} & \multirow{2}{*}{\multicolumn{2}{|c|}{$\begin{array}{l}\text { Total Cases progressing to } \\
\text { HA-MRSA Infection based } \\
\text { on initial screen result }\end{array}$}} \\
\hline & & & \multirow[t]{2}{*}{ MRSA+ } & \multirow[t]{2}{*}{ MRSA- } & & & \\
\hline & & & & & & MRSA + & MRSA - \\
\hline 2011 & 26,169 & $\begin{array}{l}24,253 \\
(92.7 \%)\end{array}$ & $\begin{array}{c}1594 \\
(6.57 \%)\end{array}$ & $\begin{array}{c}22,659 \\
(93.43 \%)\end{array}$ & $\begin{array}{c}1916 \\
(7.3 \%)\end{array}$ & 2 & 9 \\
\hline 2012 & 26,920 & $\begin{array}{l}22,074 \\
(82.0 \%) \\
\end{array}$ & $\begin{array}{c}1450 \\
(6.57 \%) \\
\end{array}$ & $\begin{array}{c}20,624 \\
(93.43 \%) \\
\end{array}$ & $\begin{array}{c}4847 \\
(18.0 \%) \\
\end{array}$ & 0 & 14 \\
\hline \multirow[t]{2}{*}{2013} & 31,688 & $\begin{array}{l}23,672 \\
(74.7 \%)\end{array}$ & $\begin{array}{c}1554 \\
(6.55 \%)\end{array}$ & $\begin{array}{c}22,118 \\
(93.45 \%)\end{array}$ & $\begin{array}{c}8016 \\
(25.3 \%)\end{array}$ & 2 & 13 \\
\hline & 84,777 & $\begin{array}{c}69,999 \\
(82.6 \%)\end{array}$ & $\begin{array}{c}4598 \\
(6.57 \%)\end{array}$ & $\begin{array}{c}65,401 \\
(93.43 \%)\end{array}$ & $\begin{array}{c}14,779 \\
(17.43 \%)\end{array}$ & $\begin{array}{c}4 \\
(82.6 \%)\end{array}$ & $\begin{array}{c}36 \\
(82.6 \%)\end{array}$ \\
\hline
\end{tabular}


Table II. Types of HA-MRSA infection based on admission MRSA screening

\begin{tabular}{lrr} 
Types of infection & $\begin{array}{r}\text { Patients not detected } \\
\text { as MRSA carriers }\end{array}$ & $\begin{array}{r}\text { Patients detected } \\
\text { as MRSA carriers }\end{array}$ \\
\hline Bacteremia & Total $\mathbf{1 6}$ & Total 4 \\
Primary & 7 & 2 \\
Thrombophlebitis/CVL*-related & 4 & 2 \\
With Joint Infection & 2 & \\
With Wound Infection & 2 & \\
With post-op infected pancreatic fluid & 1 & 0 \\
\hline Wound/Soft tissue Infection & 9 & 0 \\
\hline Bone \& Joint Infection & & 0 \\
Implant-related & 6 & 0 \\
\hline Pneumonia & 1 & 0 \\
\hline Urinary tract Infection & 2 & 4 \\
\hline Intra-abdominal Infection & 1 & \\
\hline Total & 36 & \\
\hline
\end{tabular}

*Central venous line

on entry and as mandated by hospital infection control policy all these cases were to receive daily $\mathrm{CHG}$ baths throughout their hospitalization. Compliance to the policy is high and thus the great majority of patients followed this intervention. Only 4 cases $(0.087 \%)$ among this cohort developed HA-MRSA infection.

5391 cases who either tested MRSA-negative or who missed screening are estimated to have been colonized with MRSA either through false-negative entry screening or through in-hospital acquisition. As per hospital infection control policy these cases did not receive daily $\mathrm{CHG}$ baths during hospitalization. 36 cases $(0.67 \%)$ among this estimated cohort developed HA-MRSA infection. Only 5/36 (13.9\%) were Intermediate and Long-Term Care (ILTC) sector patients. For these 36 cases the mean length of stay (LOS) until HA-MRSA infection occurred was 33.6 days (range 3-112 days; standard deviation (SD) 30.2 days). The mean overall LOS for these 36 cases was 55.8 days (range 11-144 days; SD 33.7 days)

Based on these findings the odds ratio (OR) for developing HA-MRSA infection for MRSA-colonized patients who receive daily $\mathrm{CHG}$ baths while in hospital is $0.129 ; \mathrm{P}=0.0001 ;$; $95 \%$ confidence interval
(Cl) 0.046 - 0.364; Number-needed-to-treat (NNT) =172). Another way of stating this is that for MRSAcarriers there is nearly an $87 \%$ reduction in HA-MRSA infections when daily $\mathrm{CHG}$ baths are being given.

To further highlight the protective effect of daily $\mathrm{CHG}$ baths we present the data showing the contribution of the Intermediate and Long-Term Care (ILTC) sector to the overall MRSA-carriage burden as detected in our hospital (Table III).

\section{Discussion}

To our knowledge this is only the third study to date which has looked at the impact of daily $\mathrm{CHG}$ bathing on preventing hospital-acquired infections in General Ward patients. The study by Rupp et al. ${ }^{4}$ reported a significant reduction in Clostridium difficile infections using hospital-wide CHG bathing. They employed a different approach from ours. Their study compared baseline rates of HAI's to those observed during an initial period of $\mathrm{CHG}$ bathing limited to three times per week, followed by a period where daily CHG bathing was used. There was a final washout period where CHG bathing was not used. C. difficile infections declined significantly during the $\mathrm{CHG}$ intervention period but rose significantly once $\mathrm{CHG}$ bathing was 
Table III. ILTC* Sector Contribution to MRSA Carriage in 2011, 2012 and 2013

\begin{tabular}{lrrrrrrr} 
& $\mathbf{2 0 1 1}$ & $\mathbf{2 0 1 1}$ & $\mathbf{2 0 1 2}$ & $\mathbf{2 0 1 2}$ & $\mathbf{2 0 1 3}$ & $\mathbf{2 0 1 3}$ & Totals \\
& Old & New & Old & New & Old & New & \\
\hline No. Cases & 186 & 321 & 209 & 243 & 214 & 274 & $\mathbf{1 4 4 7}$ \\
Patient days & 3276 & 4615 & 3736 & 3302 & 4057 & 3571 & $\mathbf{2 2 , 5 5 7}$ days \\
Average LOS (days) & 17.6 & 14.4 & 17.9 & 13.6 & 19.0 & 13.0 & $\mathbf{1 5 . 6}$ days
\end{tabular}

1447 cases out of total 4598 MRSA screen-positive cases detected $=31.5 \%$ from ILTC Sector

Old = cases with previously known MRSA

New = cases with no previously known MRSA

*Cases from the ILTC (Intermediate and Long-Term Care) sector include those transferred from Nursing Homes

$(1120$ cases $=77.4 \%)$, Community (Rehabilitation) Hospitals (233 cases $=16.1 \%)$, and long-term residents from the Institute of Mental Health (94 cases $=6.5 \%$ )

stopped. VRE colonization and infection rates did decline significantly during the intervention period but did not rebound when $\mathrm{CHG}$ bathing stopped. They comment that because the overall rates of HAI were low a statistically significant reduction in VRE and MRSA infections could not be demonstrated. The study by Kassakian et al. ${ }^{5}$ compared hospital-acquired MRSA,VRE, and C. difficile infection rates for all medical admissions over two sequential time periods of about one-year each. During the first time period only daily bathing with soap and water was used. During the second time period daily $\mathrm{CHG}$ bathing was used. A significant reduction was demonstrated for HA-MRSA and VRE infections but not for HA-C. difficile infections during the interval when daily $\mathrm{CHG}$ bathing was employed.

Our approach involves a retrospective analysis of three years of accumulated data looking specifically at HA-MRSA rates in two cohorts as described above. Our OR calculation depends on four variables, three of which are easily measured and one of which is a reliable estimate. The measured variables are: a) total MRSA-colonized cases detected on admission $=4598$; b) total MRSA-entry-positive cases who developed HA-MRSA infection $=4$; c) total MRSA-entry-negative cases who developed HA-MRSA infection $=36$. The one variable that is estimated is the number of cases with undetected MRSA-carriage. These are the cases that are at risk for progression to HA-MRSA infection. This estimated number provides the denominator for calculating the infection rate for our second cohort and is about 5391 cases. Even if this number were underestimated it would have to be greater than 14,746 cases at which point $\mathrm{OR}=0.36$ and the $P$ value would be $>0.05$ and no longer statistically significant. This would mean that $18.4 \%(14,746 /(84,777$ - 4598)) of all our MRSA-entry-negative cases would have to acquire MRSA while in hospital, which is clearly not the case. If this were happening we would be seeing our entry-positive MRSA prevalence steadily rising. Indeed over the past 3 years our MRSA-entry-positive rates are very stable with little variation from the overall monthly average of $6.57 \%$ (SD=0.93, Variance $=0.87$ ). Hence we can be quite confident that our results are indeed significant.

Our data demonstrate that the simple strategy of daily CHG bathing for MRSA-colonized patients throughout their hospital stay renders a significant preventive effect on HA-MRSA infections in this group. This effect is all the more impressive given that this is a highrisk cohort. Indeed 1447 (31.5\%) cases of the total of 4598 detected MRSA-carriers came from another institution classified as an Intermediate or Long-Term Care (ILTC) facility. Many of these patients (especially the ones from Nursing Homes which made up $77.4 \%$ of the cases from the ILTC Sector) have multiple comorbidities and require long-term nasogastric feeding tubes and/or long-term urinary catheters. The average length-of-stay (LOS) of this group of 1447 ILTC sector patients in fact was 15.6 days, nearly 3 times longer than the overall average LOS of 5.6 days for our entire patient population. 


\section{Figure 1. Estimating the Number of undetected MRSA Carriers}

(patients who were thus at risk for HA-MRSA Infection)

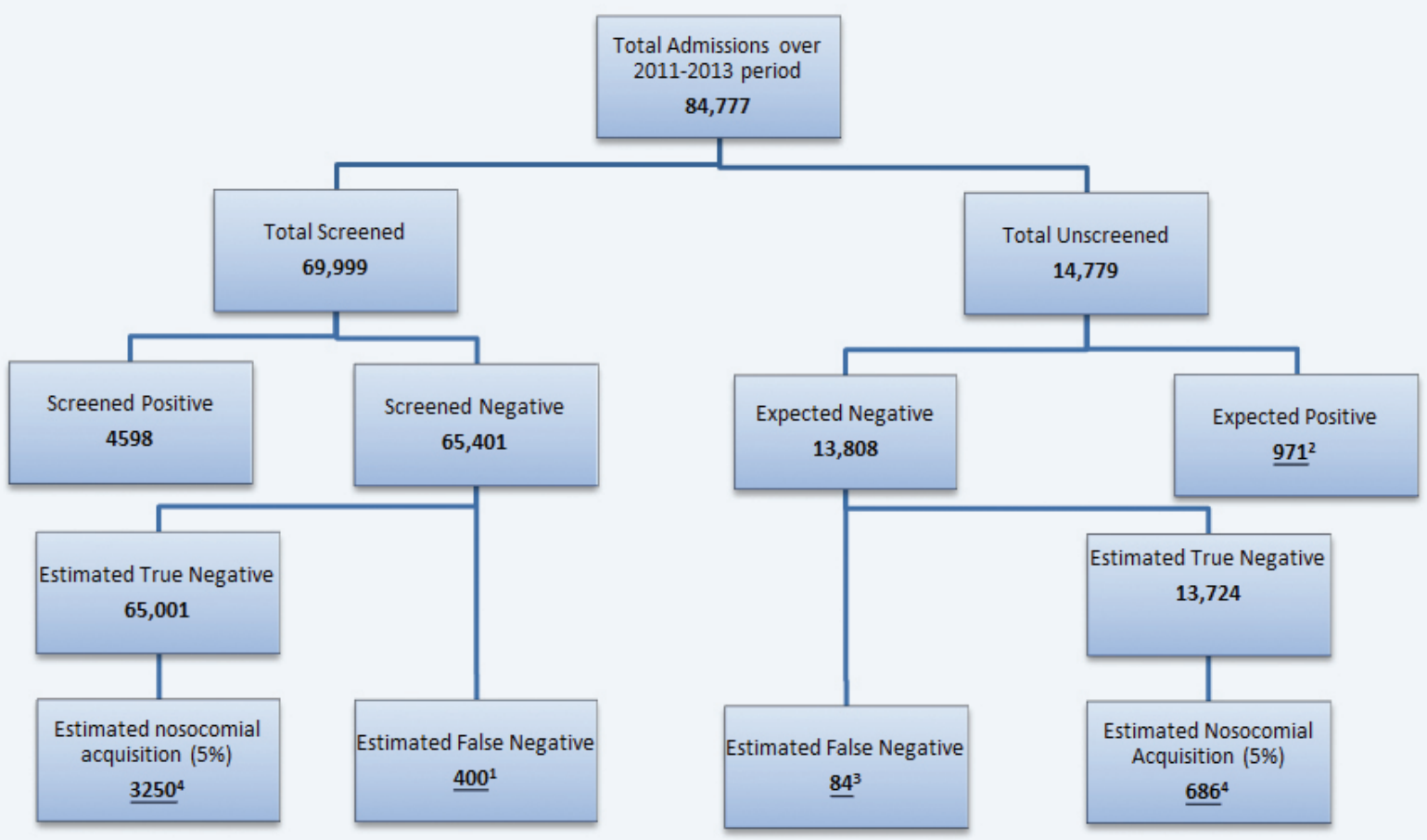

\section{Explanation of Figure 1}

1. Detection sensitivity for screening test is $92 \%$ which picked up 4598 cases over 3 years out of 69,999 cases screened. Therefore $4598=0.92 X$ where $X$ is the actual total of positive cases. $X=4998$ and missed cases (false negatives) are therefore estimated to be $\mathbf{4 0 0}(4998$ - 4598).

2. Of 14,779 unscreened cases $6.57 \%$ (our average annual positivity rate which has shown little variation from month to month and year to year) would have been expected to test positive, yielding 971 undetected cases.

3. Of unscreened cases we can estimate those who would have had a false negative test: $971=0.92 X$ where $X$ is the true total of positive cases. Therefore $X=1055$ and missed cases would be 84 (1055 - 971). If unscreened cases had been

4. $5 \%$ acquisition rate for true negatives $=3250$ $+686=3936$. True negatives are calculated as follows:

a. 65,401 screened negative minus 400 estimated to be false negatives $=65,001$ true negatives. About $5 \%$ acquisition rate gives $65,001 \times 0.05=\mathbf{3 2 5 0}$

b. Of 14,779 unscreened about 971 would have tested positive +84 estimated to test false negative $=1055$. We can estimate $14,779-1055=13,724$ true negatives. About $5 \%$ acquisition rate gives $13,724 \mathrm{x}$ $0.05=\mathbf{6 8 6}$

Grand Total of undetected MRSA carriers is $\mathbf{5 3 9 1}$ $(400+3250+971+84+686=5391)$ tested we would expect true negatives therefore to be 13,724 cases $(13,808-84)$. 
The reason for the observed protective effect of daily CHG bathing for MRSA-colonized patients is likely the reduction in overall MRSA load that any particular individual is carrying. This antimicrobial effect persists for at least 24 hours because $\mathrm{CHG}$ binds strongly to proteins in the skin. ${ }^{7,8}$ As the data were not easily obtainable we did not look at the protective effect of $\mathrm{CHG}$ bathing against other organisms where skin colonization may play a role in developing infection. These may include organisms such as MSSA, Acinetobacter baumannii and VRE.

The data (Figure 1) also suggest in-hospital MRSA transmission in our setting is responsible for the majority (73\%) of unidentified in-house MRSA carriers (about 3936 out of estimated 5391 cases). Missed screening accounted for an estimated 971 cases (18\%) of unidentified carriers. Finally we estimate 484 cases $(9 \%)$ were missed due to failure of our PCR detection test (sensitivity 92\%). To address these gaps in our processes we are reinforcing basic infection control principles such as hand-hygiene compliance, contact precautions and environmental cleaning. At the same time measures are being taken to reduce the number of cases that miss entry screening.

We should be genuinely concerned by reports of MRSA strains which have developed resistance to Chlorhexidine. ${ }^{9}$ Hence, despite the apparent protective benefits from daily in-hospital CHG baths, we need to use this anti-septic agent judiciously. This is the rationale for our policy of stopping daily $\mathrm{CHG}$ baths for patients whose MRSA-entry-screen comes negative. Nevertheless it would seem reasonable to extend the protective effects of daily CHG bathing to a wider group of patients deemed to be at high-risk for MRSA acquisition. For example, a risk-prediction tool ${ }^{10}$ for MRSA-carriage in the form of a checklist can be applied to all patients on admission. Those above a threshold score would receive daily $\mathrm{CHG}$ baths regardless of MRSA carrier status. MRSA acquisition is also closely associated with a patient's LOS in hospital. Hence another group to be targeted is long-stayers.

\section{References}

1. O'Horo JC, Silva GL, Munoz-Price LS, Safdar N. The efficacy of daily bathing with chlorhexidine for reducing healthcareassociated bloodstream infections: a meta-analysis. Infect Control Hosp Epidemiol 2012; 33: 257-267. http://dx.doi. org/10.1086/664496

2. Climo MW, Yokoe DS, Warren DK, et al. Effect of daily chlorhexidine bathing on hospital-acquired infection. N Engl / Med 2013; 368: 533-542. http://dx.doi.org/10.1056/NEJMoa1113849

3. Climo MW, Sepkowitz KA, Zuccotti G, et al. The effect of daily bathing with chlorhexidine on the acquisition of methicillin-resistant Staphylococcus aureus, vancomycinresistant Enterococcus, and healthcare-associated bloodstream infections: results of a quasi-experimental multicenter trial. Crit Care Med 2009; 37(6): 1858-1865. http://dx.doi.org/10.1097/CCM.0b013e31819ffe6d

4. Rupp ME, Cavalieri RJ, Lyden E, et al. Effect of hospitalwide chlorhexidine patient bathing on healthcare-associated infections. Infect Control Hosp Epidemiol 2012; 33: 10941100. http://dx.doi.org/10.1086/668024

5. Kassakian SZ, Mermel LA, Jefferson JA, Parenteau SL, Machan JT. Impact of chlorhexidine bathing on hospitalacquired infections among general medical patients. Infect Control Hosp Epidemiol 2011; 32: 238-243. http://dx.doi. org/10.1086/658334

6. Willis GC, Chng FLC, Foo ML, Ooi ST. The effect of daily chlorhexidine baths on nosocomial methicillin-resistant Staphylococcus aureus infection in general wards. In: Program and abstracts of inaugural ID Week 2012; October 16-21, 2012; San Diego, CA. Abstract 1545. https://idsa.confex.com/ idsa/2012/webprogram/Paper34533.html

7. Lim KS, Kam PC. Chlorhexidine - pharmacology and clinical applications. Anaesth intens care 2008; 36: 502-512.

8. Hibbard JS. Analyses comparing the antimicrobial activity and safety of current antiseptic agents. J Infus Nurs 2005; 28: 194207. http://dx.doi.org/10.1097/00129804-200505000-00008

9. Longtin J, Seah C, Siebert K, et al. Distribution of antiseptic resistance genes qac $A$, qacB, and $s m r$ in Methicillin-resistant Staphylococcus aureus isolated in Toronto, Canada, from 2005 to 2009. Antimicrob Agents Chemother 2011; 55: 2999-3001. http://dx.doi.org/10.1128/AAC.01707-10

10. Ooi ST, Lim SC, Chen YM, Chng FLC, Willis GC. Clinical predictors of methicillin-resistant Staphylococcus aureus colonization on admission. In: Program and abstracts of the $49^{\text {th }}$ annual meeting of the Infectious Diseases Society of America (IDSA) 2011; October 20-23, 2011; Boston, MA. Abstract 551 https://idsa.confex.com/idsa/2011/webprogram/ Paper31331.html 